\title{
Challenges in optimizing preexposure prophylaxis development, engagement, and access for HIV prevention
}

\author{
Eileen P. Scully, ${ }^{1}$ Ethel D. Weld, ${ }^{1}$ and Joel N. Blankson ${ }^{1,2}$ \\ 'Department of Medicine and 2Department of Molecular and Comparative Pathobiology, The Johns Hopkins University School of Medicine, Baltimore, Maryland, USA.
}

0 ne of the greatest successes of modern science is the development of effective antiretroviral therapy (ART) for control of HIV-1 infection. Arguably one of the more innovative responses to the ongoing HIV epidemic is the repurposing of ART for preventive efforts as preexposure prophylaxis (PrEP). This strategy has been critical in the face of approximately 1.7 million new infections in 2018 and in the absence of an effective HIV vaccine. Notwithstanding this advance, significant scientific challenges remain for the optimal implementation of PrEP, and novel approaches are still needed (1).

In the United States, currently approved PrEP consists of a single pill containing two antiretroviral drugs taken daily. While this regimen substantially reduces the risk of HIV-1 acquisition, PrEP has still not reached the majority of at-risk individuals, even in resource-rich settings (2). Comprehensive preventive services are a cornerstone of the Department of Health and Human Services' strategic plan to end the HIV epidemic (3), and PrEP is the biomedical intervention currently available. Preventive interventions are at the intersection of medical and behavioral science, and their rollout can highlight disparities in health care access. PrEP is no different, requiring rigorous efforts to achieve its potential equitably across diverse groups.

We propose that PrEP offers a window into the efforts to end the HIV epidemic while also illuminating the tension between innovation in biomedical science and its application in public health efforts.

\section{How effective is PrEP and for whom?}

PrEP efficacy was initially demonstrated among men who have sex with men
(MSM) with high-risk sexual behavior, reducing risk of HIV acquisition versus placebo (relative risk reduction [RRR] of $44 \%$ ), with even greater protection (89\% RRR) in a subgroup with confirmed presence of the study drug in blood (4). Similar results were seen in serodiscordant couples in which the partner with HIV was not on ART and the uninfected partner was randomized to receive PrEP: an overall RRR of $75 \%$ with PrEP, and 90\% RRR with detectable drug levels (5).

Of note, initial studies done exclusively in women at risk of sexual acquisition were not as successful $(6,7)$; the apparent failures were driven predominantly by very low adherence (8). More recent studies have helped to clarify that PrEP is indeed effective in women, although because of differences in tissue site drug levels and other factors, daily adherence may be required to achieve full protection (8). Direct interactions between the vaginal microbiome and tenofovir may impact efficacy at least for topical therapy approaches (9), which suggests the need for caution when extrapolating data between populations. These concerns are highlighted by the recent FDA approval of a second drug for PrEP, emtricitabine/tenofovir alafenamide; this approval excludes women with vaginal receptive sex as their risk, as the formulation has not been studied in women. In sub-Saharan Africa, four in five new infections among adolescents aged 15-19 years are in girls, therefore appropriate testing and validation in women, and optimization of delivery and uptake are paramount (10). Testing PrEP in the context of conception, pregnancy, and the postpartum period is also important for meaningful risk mitigation, particularly given the high risk of

Conflict of interest: The authors have declared that no conflict of interest exists.

transmission to the infant in the context of acute infection during pregnancy (11). Implementation and demonstration projects have highlighted that PrEP must be acceptable and accessible to people who need it; scientific demonstration of efficacy is inadequate to achieve full impact (12).

PrEP is also highly effective in preventing transmission via injection drug use (13), although implementation of PrEP in this population is minimal. This deficit in the use of PrEP is particularly relevant in regions where adjunctive harm reduction strategies (e.g., needle exchanges) are inadequate and drug use is highly criminalized. A cluster of HIV transmission through injection in the United States highlights the potential for an intersection between the opioid and HIV epidemics and the need to offer preventive options (3).

\section{What are the risks of PrEP?}

As with any medication, there is a small risk of idiosyncratic drug reaction and a risk of adverse effects, including modest declines in bone mineral density or renal impairment. However, with real-world implementation of PrEP, these risks have proven to be minimal (14). There are also scattered case reports of PrEP failure and a lingering concern for inadvertent initiation of PrEP in individuals already infected with HIV, but this has been a rare phenomenon overall. Perhaps a greater detriment to the adoption of PrEP has been a perceived risk that PrEP could lead to a rise in sexually transmitted infections (STIs) due to risky sexual practices. Increased STI incidence has been seen in a subset ( $25 \%)$ of PrEP users, but this observation needs to be viewed in the context of increased frequency of testing for STIs $(15,16)$. The clinical decision-making impact of a perceived risk compensation must be directly addressed, as it is likely to bias providers away from PrEP prescription $(17,18)$. 
A
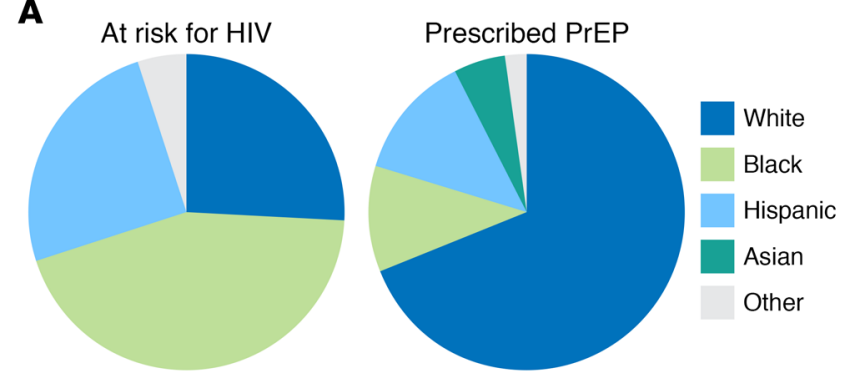

B
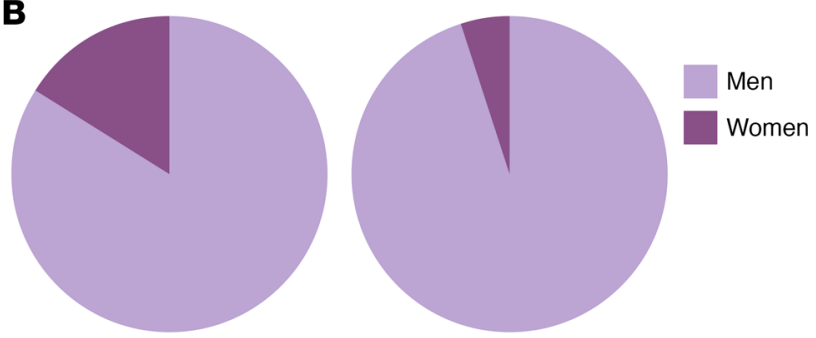

Figure 1. HIV infection risk and PrEP prescriptions in the United States. (A) Estimated proportions of people living in the United States at risk for HIV infection (2015 data) and distribution of PrEP prescriptions in 2016 among the subset for whom race/ethnicity data is available. (B) Risk versus prescription frequency among men and women (2015 data). Figure based on data in refs. 19, 21.

\section{What is limiting the effectiveness of PrEP?}

A 2019 study by the CDC examined the impact of targeted outreach to MSM about PrEP, with PrEP awareness increasing from $60 \%$ to $90 \%$ from 2014 to 2017 (2). However, despite this rise in awareness, active use of PrEP remained limited, increasing from $6 \%$ to $35 \%$ of eligible individuals over the same period. There is a notable racial disparity in PrEP uptake, with Black MSM using PrEP at approximately half the rate of White MSM (Figure 1). Moreover, PrEP use is also linked to higher educational attainment, income, and insurance status (2).

Of note, PrEP education efforts have primarily engaged MSM. Transgender women, who have a disproportionate risk of HIV infection, often have limited engagement with health care services. In the United States, women at risk for HIV infection are unlikely to self-identify or to be assessed to be at risk of HIV by health care providers. PrEP use among women has remained very low and static, at less than $5 \%$ of prescriptions and $2 \%$ of the women at risk during a period of expanding use among MSM (19). The best ways of reaching women at risk remains a topic of active investigation.

In addition to the challenges of identifying and engaging people at risk for HIV, continued adherence to the PrEP regimen is required. Recent studies have demon- strated that high levels of initial adherence are often not sustained over time (12).

Future strategies that address this challenge may include event-driven prophylaxis, proven effective among MSM (20); longer-acting formulations of PrEP; behaviorally congruent delivery methods; and other novel drugs and formulations (reviewed in ref. 21). A single PrEP modality, as is currently available, is unlikely sufficient for broad population-based impact, and consistent with the experience of contraception, offering a choice among an array of acceptable methods is likely to be a major driver of improved uptake (1).

\section{Can PrEP interrupt the HIV epidemic?}

Outside the rarefied setting of a clinical trial, PrEP has shown high preventive efficacy, and, in some cases, implementation and demonstration projects have shown decreases in local HIV incidence. Together with early treatment to prevent transmission (treatment as prevention) and in an era when patients on ART with undetectable viral loads have been shown not to transmit the virus (undetectable $=$ transmissible), PrEP can bring us closer to the goal of ending the HIV epidemic, which has already claimed 35 million lives (3). However, even with more diverse implementation options, PrEP is not likely to be the only solution to the problem. As seen in the hepatitis $\mathrm{C}$ epidemic, even highly successful curative therapy will not be sufficient alone to end the epidemic, and an adjunctive vaccine would be highly impactful. Similarly, a vaccine is still urgently needed for HIV prevention. This need does not diminish the role of PrEP as an immediately available and effective intervention - one that further scientific innovation can certainly extend and improve.

\section{Conclusions}

The story of the development of PrEP is one of the astounding successes in medicine. The efficacy of this intervention has exceeded our best efforts to date at HIV vaccine design. But PrEP is also a story of barriers for both people at risk of HIV infection and providers, and an important illustration of how interventions are only successful when they can be effectively implemented. We would also argue that PrEP highlights the critical need for innovation from basic scientists and clinicians even after an initial "solution" has been achieved $(1,21)$. Although currently available PrEP is efficacious, the challenges of adherence and access emphasize the need for novel approaches to deliver therapies in ways that are acceptable, feasible, and available to the individuals at risk of HIV infection. Some of these strategies were highlighted at the recent 10th International AIDS Society Conference on HIV Science in Mexico City, and the recent call for new investigation in this arena from the NIH is an opportunity to expand and develop the science of prevention. Now more than ever, physicians and scientists have a critical role in developing new approaches, advocating for effective interventions, and translating the best possible science to at-risk populations as well as to providers and policy makers in order to realize the optimal benefits of PrEP.

\section{Acknowledgments}

We thank Craig Hendrix and Guido Massacessi for thoughtful comments on a draft of this article.

Address correspondence to: Joel N. Blankson, 855 N Wolfe Street, Rangos 822, Baltimore, Maryland 21205, USA. Phone: 410.502.9920. Email: jblanks@jhmi.edu. 
1. Hendrix CW. HIV Antiretroviral pre-exposure prophylaxis: development challenges and pipeline promise. Clin Pharmacol Ther. 2018;104(6):1082-1097.

2. Finlayson T, et al. Changes in HIV preexposure prophylaxis awareness and use among men who have sex with men -20 urban areas, 2014 and 2017. MMWR Morb Mortal Wkly Rep. 2019;68(27):597-603.

3. Fauci AS, Redfield RR, Sigounas G, Weahkee MD, Giroir BP. Ending the HIV epidemic: a plan for the United States. JAMA. 2019;321(9):844-845.

4. Grant RM, et al. Preexposure chemoprophylaxis for HIV prevention in men who have sex with men. N Engl JMed. 2010;363(27):2587-2599.

5. Baeten JM, et al. Antiretroviral prophylaxis for HIV prevention in heterosexual men and women. N Engl JMed. 2012;367(5):399-410.

6. Marrazzo JM, et al. Tenofovir-based preexposure prophylaxis for HIV infection among African women. N Engl J Med. 2015;372(6):509-518.

7. Van Damme L, et al. Preexposure prophylaxis for $\mathrm{HIV}$ infection among African women. $\mathrm{N} \mathrm{Engl} \mathrm{J}$ Med. 2012;367(5):411-422.

8. Sheth AN, Rolle CP, Gandhi M. HIV preexposure prophylaxis for women. J Virus Erad. 2016;2(3):149-155.

9. Klatt NR, et al. Vaginal bacteria modify HIV tenofovir microbicide efficacy in African women. Science. 2017;356(6341):938-945.

10. [No authors listed]. Global HIV \& AIDS statistics -2019 fact sheet. UNAIDS. https://www. unaids.org/en/resources/fact-sheet. Accessed November 4, 2019.

11. Matthews LT, et al. Protocol for a longitudinal study to evaluate the use of tenofovir-based PrEP for safer conception and pregnancy among women in South Africa. BMJ Open. 2019;9(7):e027227.

12. Celum CL, et al. HIV pre-exposure prophylaxis for adolescent girls and young women in Africa: from efficacy trials to delivery. J Int AIDS Soc. 2019;22(suppl 4):e25298.

13. Choopanya K, et al. Antiretroviral prophylaxis for HIV infection in injecting drug users in Bangkok, Thailand (the Bangkok Tenofovir Study): a randomised, double-blind, placebo-controlled phase 3 trial. Lancet. 2013;381(9883):2083-2090.

14. Marcus JL, et al. Preexposure prophylaxis for HIV prevention in a large integrated health care system: adherence, renal safety, and discontinuation. J Acquir Immune Defic Syndr. 2016;73(5):540-546.

15. Beymer MR, et al. Does HIV pre-exposure prophylaxis use lead to a higher incidence of sexually transmitted infections? A case-cross- over study of men who have sex with men in Los Angeles, California. Sex Transm Infect. 2018;94(6):457-462.

16. Traeger MW, et al. Association of HIV preexposure prophylaxis with incidence of sexually transmitted infections among individuals at high risk of HIV infection. JAMA. 2019;321(14):1380-1390.

17. Gandhi M, Spinelli MA, Mayer KH. Addressing the sexually transmitted infection and HIV syndemic. JAMA. 2019;321(14):1356-1358.

18. Marcus JL, Katz KA, Krakower DS, Calabrese SK. Risk compensation and clinical decision making - the case of HIV preexposure prophylaxis. NEngl JMed. 2019;380(6):510-512.

19. Huang YA, Zhu W, Smith DK, Harris N, Hoover KW. HIV preexposure prophylaxis, by race and ethnicity - United States, 2014-2016. MMWR Morb Mortal Wkly Rep. 2018;67(41):1147-1150.

20. Molina JM, et al. Efficacy, safety, and effect on sexual behaviour of on-demand pre-exposure prophylaxis for HIV in men who have sex with men: an observational cohort study. Lancet HIV. 2017;4(9):e402-e410.

21. Beymer MR, Holloway IW, Pulsipher C, Landovitz RJ. Current and future PrEP medications and modalities: on-demand, injectables, and topicals. Curr HIV/AIDS Rep. 2019;16(4):349-358. 